\title{
A MEMS-RELAY FOR MAKE-BREAK POWER SWITCHING APPLICATIONS
}

\author{
A.C. Weber ${ }^{1}$, J.H. Lang $^{2}$, and A.H. Slocum
}

${ }^{1}$ Mechanical Engineering, Massachusetts Institute of Technology, Cambridge, MA, USA

${ }^{2}$ Electrical Engineering and Computer Science, Massachusetts Institute of Technology, Cambridge, MA, USA

\begin{abstract}
This paper presents the design, modeling, fabrication and testing of a horizontal-displacement, electrostatically-actuated, MEMS relay for make-break power switching applications. The relay features $\{111\}$-plane silicon-etched electrical contacts. It is etched in (100) Si through a combination of $\mathrm{KOH}$ etching and DRIE, bonded to a glass substrate, and plated with a $10 \mu \mathrm{m}$ thick copper, and a $2 \mu \mathrm{m}$ thick palladium-cobalt film. Experimental relays exhibit a minimum total on-state contact resistance of $130 \mathrm{~m} \Omega$, a response time of $750 \mu \mathrm{s}$, a theoretical electrical isolation in excess of $1 \mathrm{kV}$ (tested to $450 \mathrm{~V}$ with available equipment), and a current carrying capacity of $800 \mathrm{~mA}$. They have been hot-switched in excess of $10^{5}$ cycles without signs of performance degradation.
\end{abstract}

\section{INTRODUCTION}

MEMS relays and switches are of interest in applications such as test equipment, radars, communications and power systems, amongst others [1, 2]. Unlike solid state devices, mechanical relays provide galvanic isolation between the control and power ports. Heat dissipation and tribology impose significant constraints on MEMS relays which typically operate at contact forces on the order of $\mu \mathrm{N}$ to $\mathrm{mN}[1,2]$. Telecom relays, for example, may be subject to surges of up to $2.5 \mathrm{kV}$ and several Amperes [3]. The purpose of this research is to develop a MEMSrelay capable of reliably hot-switching currents on the order of $1 \mathrm{~A}$, and providing galvanic isolation in excess of $1 \mathrm{kV}$.

\section{DESIGN AND MODELING}

In order to achieve reliable operation, high on-state current carrying capacity, high off-state breakdown voltage, and the capability of reliably hot-switching resistive and inductive loads, the following functional requirements must be met.

- Contact travel must exceed $10 \mu \mathrm{m}$ to prevent arcing while operating in air at atmospheric pressures, as expressed by Paschen's law $[4,5]$. Contact travel over $30 \mu \mathrm{m}$ is preferred for hot-switching, as contact erosion may lead to bridging [6].

- A minimum closing force of $0.1-0.6 \mathrm{mN}$ is required to achieve stable contact performance, and $5-10 \mathrm{mN}$ is required to achieve low contact resistance with hard metals such as $\mathrm{Ru}$ [7].

- A minimum opening force of $0.1-2.7 \mathrm{mN}$ is required to overcome contact adhesion [7].

- Contact wipe is desired to increase the reliability of the contacts by removing oxide and organic films.

- Adequate contact materials and metallization processes are desired, to provide stable and low contact-resistance, prevent material transfer, and achieve high arc voltages and currents. Materials with high melting and boiling temperatures are preferred [3].

\section{Device Architecture}

Based on the functional requirements, we designed a single pole, single-throw, serial metal-contact MEMS relay. This relay, shown in Figure 1, is comprised of four double-parallelogram flexures (1) which serve as bearings, eight pairs of engaging and disengaging electrostatic "zipper" actuators (2), one moving $\{111\}$ contact (3), and a pair of static $\{111\}$ contacts (4a, 4b).
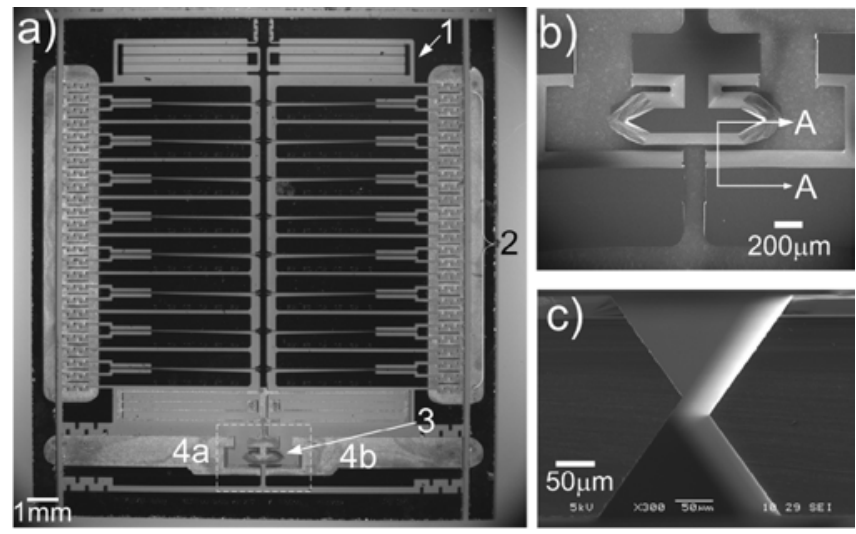

Figure 1: Fabricated relay. Die top view (a); contact detail, prior to metal deposition (b); contact cross section $A-A$, as shown in Figure $1 b$, without the metal film (c).

\section{\{111\} Silicon Etched Contacts}

The $\{111\}$-plane contacts offer several advantages over traditional MEMS-relay metal contacts such as vertical displacement contacts (VDC) and horizontal displacement contacts (HDC). VDC, shown in Figures 2a and 2b, have a contact travel on the order of $1-5 \mu \mathrm{m}[1,2]$, which is less than the $30 \mu \mathrm{m}$ required to withstand contact erosion [6] and the $10 \mu \mathrm{m}$ required to prevent arcing $[4,5]$. Traditional HDC, shown schematically in Figures 2c and 2d, have resistances on the order of 1-10 Ohms [8, 9], which leads to excessive Joule heating while handling the currents in our application; further it is difficult to deposit metal onto these contacts, making continuity challenging. The $\{111\}$ contacts [10], shown schematically in Figures 2e and 2f, provide large travel, on the order of $70 \mu \mathrm{m}$, which prevents arcing, and introduces contact wipe which is known to enhance the contact reliability. Further, the oblique geometry allows for an enhanced metallization process which provides low on-state contact resistance values.
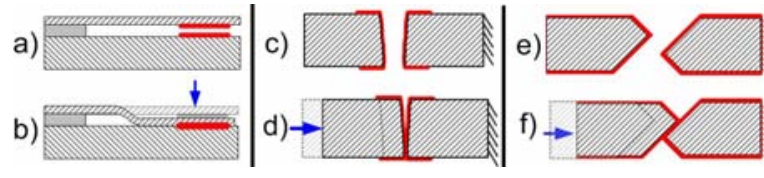

Figure 2: Contact architecture. Vertical displacement contacts (VDC) in open (a) and closed (b) states; horizontal displacement contacts (HDC) in open (c) and closed(d) states; $\{111\}$ Si etched contacts in open (e) and closed ( $f$ ) states.

\section{Actuation}

Eight pairs of compliant-starting-zone zipper (CSZZ) actuators [11] provide a theoretical force of $40 \mathrm{mN}$ during the closing and opening events, a pull-in voltage of $65 \mathrm{~V}$ and a $70 \mu \mathrm{m}$ travel. Electrostatic actuation was chosen due to its low, virtually negligible, power consumption and heat generation; CSZZ actuators were selected to reduce the pull-in voltage, while providing the required displacement and force. 


\section{Contact Material}

The $\{111\}$-plane contacts were electroplated with $10 \mu \mathrm{m}$ of copper $(\mathrm{Cu})$ and $2 \mu \mathrm{m}$ of palladium-cobalt (Pd-Co) over an evaporated gold $(\mathrm{Au})$ seed layer. Pd was selected for its low and stable contact resistance, robustness to material transfer, high minimum arc voltages and arc currents, high melting and boiling temperatures; the alloying material Co was selected to increase the hardness of the Pd alloy. However, it was found during testing that the Co readily oxidized in the presence of an arc, thus pure Pd could yield better results than those presented here.

\section{FABRICATION}

The MEMS relay fabrication process, shown in Figure 3, proceeds as follows: the device layer is etched in (100) Si through a combination of deep-reactive-ion-etching (DRIE) and crystallineorientation-dependant etching with $\mathrm{KOH}$ using nested silicondioxide and silicon-nitride masks (a-k); a Au seed layer is evaporated through a shadow wafer onto both sides of the device wafer (l); the device layer is bonded to a glass substrate (m); the conductive metal films are electroplated over the seed-layer onto the contacts; the device is released by dicing through the device layer; finally, the device is wire-bonded to a pin grid array IC package for testing.

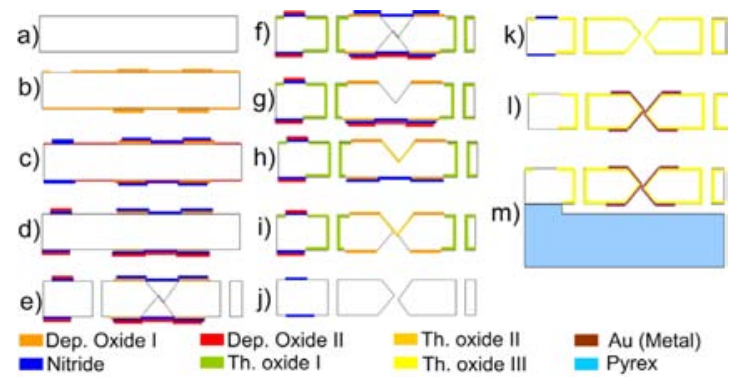

Figure 3: Fabrication process.

\section{EXPERIMENTAL RESULTS}

During testing, voltages and currents are continuously monitored as the relay cycles, and the instantaneous total contact resistance is computed, as shown in Figures 4 and 5.

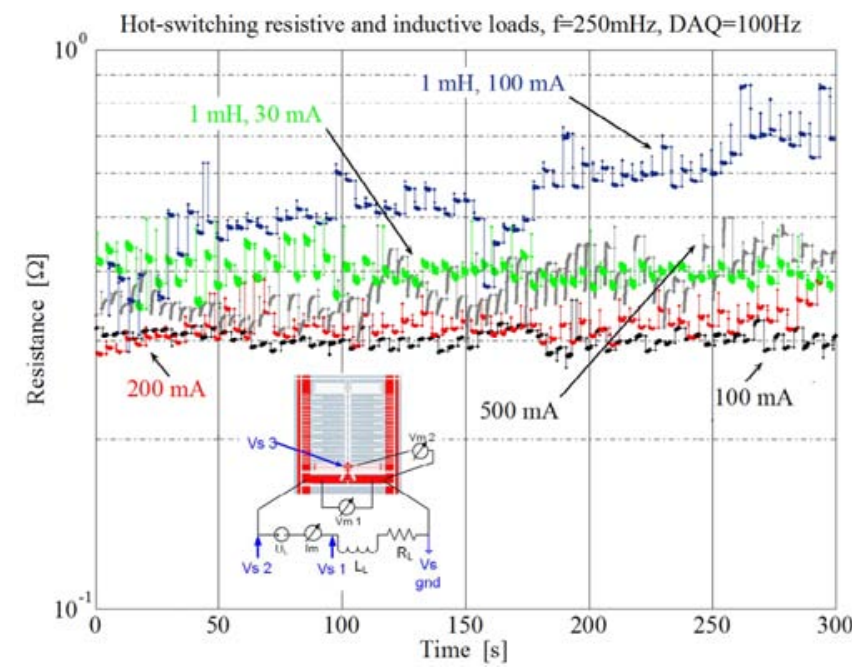

Figure 4: Continuous sampling of total contact resistance during hot-switching. Inset: Experimental setup.

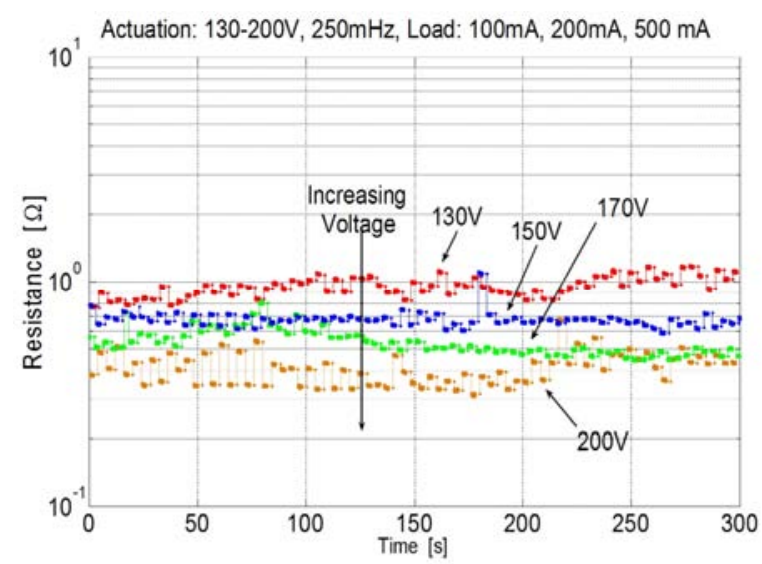

Figure 5: Total contact resistance at various actuation voltages.

The test setup is shown in the inset of Figure 4. Voltage and current are sampled continuously and synchronously via multimeters (Im, Vm1, Vm2), while an oscilloscope is used for the transient response (Vs1, Vs2, Vs3).

The load current and voltage were increased until the relay showed any signs of temporary contact-sticking during any actuation cycle. The sticking was found to be reversible, the contacts recovered after cycling without the load connected. Thus no permanent damage was caused to the contact surfaces. The maximum hot-switched current achieved without any signs of contact sticking was $800 \mathrm{~mA}$ with a resistive load and $350 \mathrm{~mA}$ with a $1 \mathrm{mH}$ inductive load. The integrity of the contact surfaces was attested with a scanning electron microscope before and after hot-switching.

\section{CONCLUSIONS}

The MEMS relay exhibits theoretical isolation in excess of $1 \mathrm{kV}$ (tested to $450 \mathrm{~V}$ with available equipment), repeatable and reliable hot-switching characteristics with load currents up to $800 \mathrm{~mA}$ and $350 \mathrm{~mA}$ with resistive and inductive loads respectively. The relay was tested over $10^{5}$ cycles. The Pd-Co film was found to readily oxidize in the presence of an arc and during hot temperature processing in an oxidizing environment. In order to improve device performance, the Pd-Co film should be replaced by pure Pd or by a refractory metal.

\section{ACKNOWLEDGMENT}

Microfabrication was performed at the Microsystems Technology Laboratories at MIT. The electroplating was performed at FormFactor Inc., 7005 South Front Rd., Livermore, CA 94551. The authors thank Dr. Mike Armstrong and Dr. Rod Martens for their assistance and the insightful discussions.

\section{REFERENCES}

[1] G.M. Rebeiz, RF MEMS theory design and tech., JW\&S '03.

[2] J.J. Yao, J. Micromech. Microeng., Vol. 10, '00, pp. R9-R38.

[3] W. Johler, IEEE TCPT, 27(1), March '04, pp. 19-29.

[4] J.-E. Wong, et. al. MEMS 2000, Japan, ’00, pp. 633-638.

[5] H. Hosaka, et. al., Sens. and Act. A., (40-1), Jan '94, pp. 41-47.

[6] S.T. Patton, et. al., Tribology Lett., 18-2, Feb '05, pp. 215-230.

[7] J. Schimkat, Sensors and Actuators, 73(1999), pp. 138-143

[8] J. Qiu, et. al., JMEMS, Vol. 14(5), Oct. 2005, pp. 1099-1109

[9] W.P. Taylor, et. al., JMEMS, Vol. 7(2), 1998, pp. 181-191.

[10] A.C. Weber, et. al., 53 ${ }^{\text {rd }}$ Holm Conf., Sept. '07, pp. 156-159.

[11] J. Li, et. al., JMEMS, Vol. 14(6), 2005, pp. 1283-1297. 